\title{
Proton Damage in Linear and Digital Optocouplers ${ }^{\dagger}$
}

\author{
A. H. Johnston, and B. G. Rax \\ Jet Propulsion Laboratory \\ California Institute of Technology \\ Pasadena, California
}

\section{Introduction}

Optocoupler failures occurred on the TopexPoseidon spacecraft after about two years of operation. Later work in the laboratory showed that the failures were due to extreme sensitivity of LEDs within the optocouplers to displacement damage from protons [1-3]. Although earlier work had been done on displacement damage in lightemitting diodes, none of the devices studied previously had been heavily damaged at the low radiation levels where the optocouplers failed in space [4-7]. Subsequent work has shown that LED damage varies over an extremely wide range, depending on the particular manufacturing technology.

This paper discusses proton degradation of linear and digital optocouplers. One obvious way to harden optocoupler technologies is to select LEDs that are more resistant to displacement damage. A direct comparison is made of degradation of a commercial linear optocoupler from one manufacturer with a modified version of the same device with a different LED technology. Other factors, including degradation of optical photoresponse and transistor gain are also discussed, along with basic comparisons of digital and analog optocouplers. Linear optocouplers are designed with somewhat different requirements than digital optocouplers, which not only affects their radiation response but also the interpretation of radiation test data.

Optocoupler degradation depends on the degradation of both III-V and silicon devices, Consequently there is some ambiguity about how to compare damage at different proton energies because the energy dependence of non-ionizing energy loss (NIEL) is different for the two materials. In addition, recent experimental data on the energy dependence of proton damage in LEDs [8] does not agree with earlier calculation of NIEL for GaAs semiconductors that was based primarily on JFETs [9], suggesting that there are unresolved issues relating to NIEL in III-V devices. We have

t The research in this paper was carried out by the Jet Propulsion Laboratory. California Institute of Technology. under contract with the National Aeronautics and Space Administration. Code AE, under the NASA Microelectronics Space Radiation Effecis Program (MSREP). chosen to do our experimental work with $50 \mathrm{MeV}$ protons, which is near the peak in the proton energy spectrum for many earth-orbiting systems with shielding thicknesses of about 100 mils of aluminum. This reduces the magnitude of adjustments to account for the energy dependence compared to the approach used for solar cell degradation ( $10 \mathrm{MeV}$ equivalent damage).

\section{Comparison of Standard and Hardened Optocouplers}

The standard optocoupler produced by this manufacturer uses a diffused LED with amphoteric doping. The degradation of a typical LED from the standard device is compared with degradation of a double-heterojunction LED that is used in the hardened optocouplers in Figure 1. The revised LED provides about an order of magnitude improvement compared to the normal LED. The phototransistor was the same in both the hardened and standard devices.

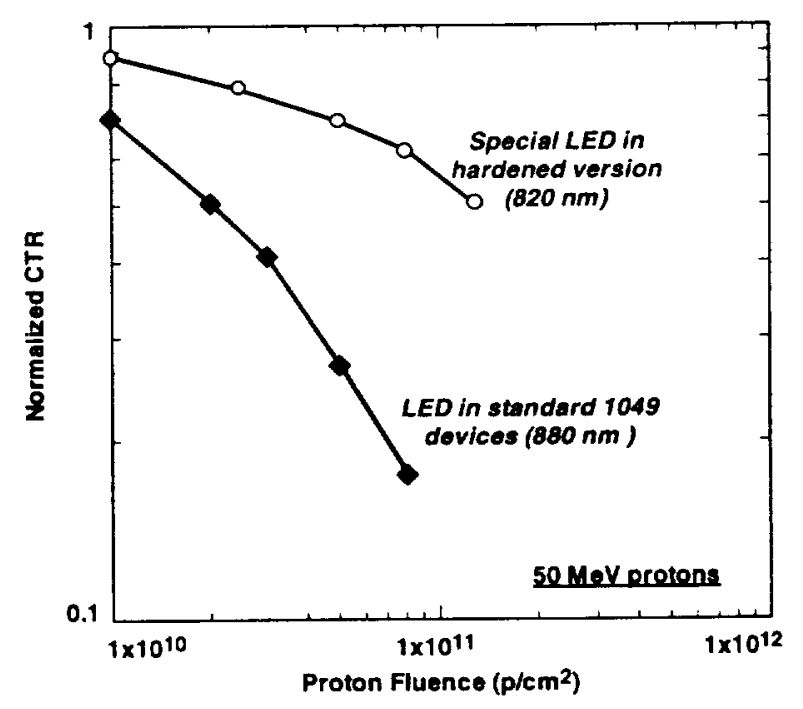

Figure 1. Degradation of the two LED technologies used in standard and hardened optocouplers.

The degradation of optocouplers fabricated with the two LED technologies is shown in Figure 2. The devices were irradiated with bias applied to the collector of the phototransistor, but with no forward current through the LED. Note the strong dependence of the degradation on the forward 
current of these analog optocouplers. Although the degree of improvement in radiation response is roughly what is expected from substitution of the LED, a closer examination of the results shows that there is actually less damage in the hardened device than expected from the difference in LED degradation alone. This is caused by the particular way in which analog optocouplers are designed to reduce both the variability in CTR between units and to "flatten" the strong negative temperature coefficient of the LED.

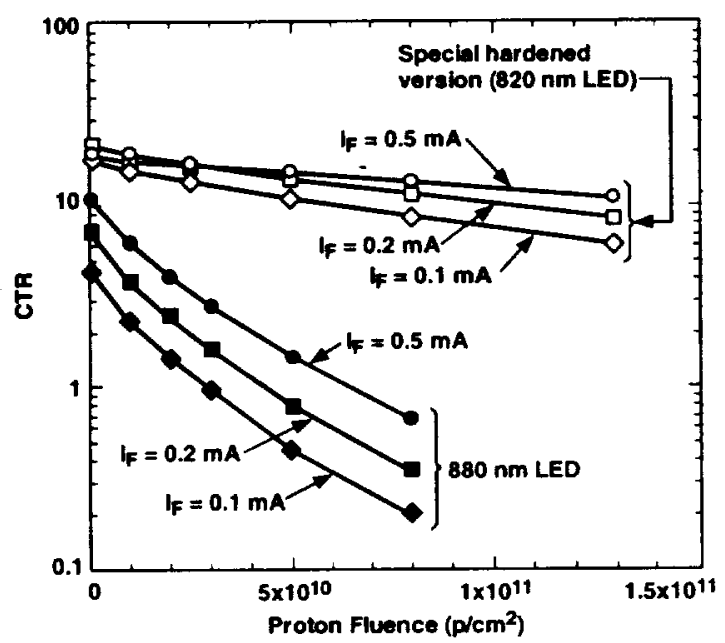

Figure 2. Degradation of standard and special versions of the OLH300 linear optocoupler.

Figure 3 shows how CTR current dependence of the unhardened optocoupler changes after irradiation (the duty cycle of the measurements was low to eliminate possible interference from

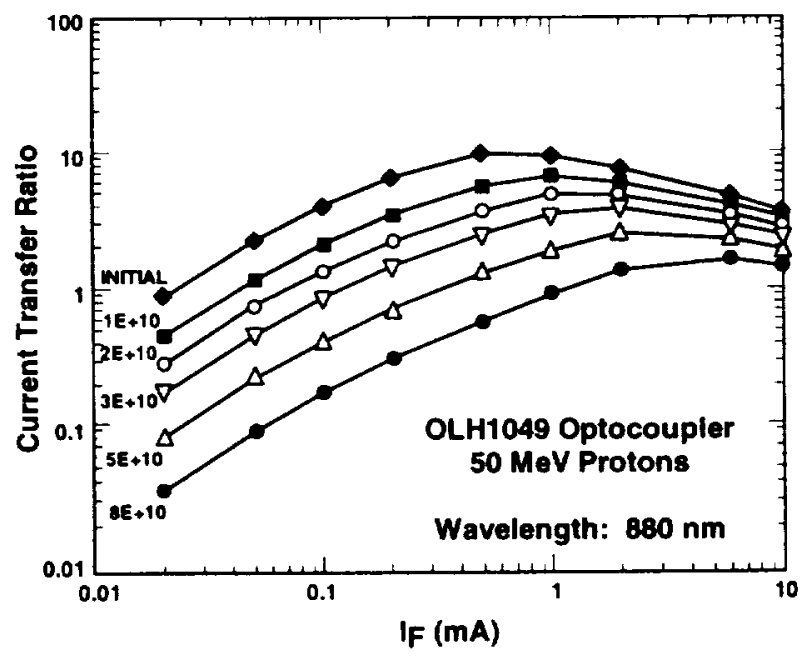

Figure 3. Degradation of the standard version of the optocoupler at various LED forward currents. current-enhanced annealing [8]). The main reason for this dependence is the current dependence of phototransistor gain. At low radiation levels the change in (optical) drive current as the LED degrades is small, so the operating point of the phototransistor remains near the flat region (with the normal range of LED currents). However, at higher radiation levels the light output of the LED is markedly reduced, and the operating point shifts to regions with steeper slope, where the falloff in CTR with current increases the amount of degradation beyond that expected from just the LED degradation.

Figure 4 shows the current dependence of CTR for the improved optocouplers. Initially these devices are deliberately operated well beyond the peak in the transistor characteristics (high injection region). This reduces the sensitivity to temperature effects in the LED and tightens the distribution of CTR. It also causes degradation in CTR to be less than the degradation in LED output because the reduced light output from the LED shifts operation to lower transistor current, where the transistor gain is higher. Thus, the particular way in which analog devices are designed tends to mask internal changes in operating conditions until the collector current falls below the peak in the operating characteristics.

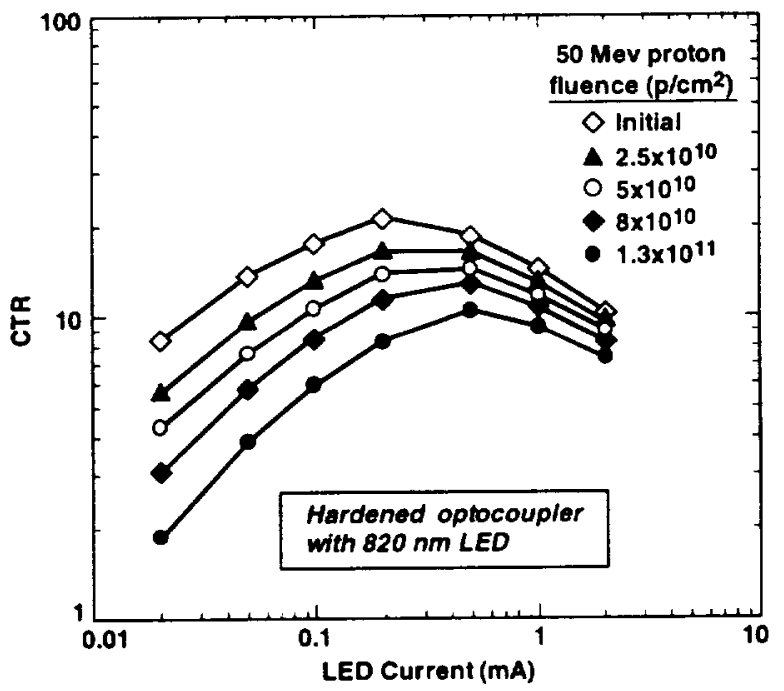

Figure 4. Degradation of the special version of the optocoupler at various LED forward currents.

As a result, linear optocouplers have a different dependence on fluence than digital optocouplers. CTR degradation in digital devices tends to track LED degradation, exhibiting large changes at low fluence levels which gradually "flatten" at higher fluences. The CTR degradation of linear optocouplers is less severe at lower radiation levels 
than that of digital optocouplers because the normal operating region of linear optocouplers is above the peak current region.

Some further comments are in order regarding LED degradation. Although only a limited number of LED types have been subjected to radiation testing, the evidence to date suggests that there are fundamental differences in the radiation sensitivity of two basic types of LEDs: diffused LEDs, which are fabricated with GaAs or AlGaAs (depending on wavelength); and double-heterojunction (DH) LEDs, which are fabricated with a more complex process that involves two or more layers of dissimilar semiconductor materials. Diffused LEDs are less costly to manufacture, and have very high efficiency near the wavelength where silicon has maximum responsivity (approximately $900 \mathrm{~nm}$ ).

Figure 5 compares the degradation of five different types of LEDs from various manufacturers (the measurement current is approximately $40 \%$ of the maximum recommended current). Note that the DH technology devices have at least an order of magnitude less degradation compared to diffused LEDs. Degradation of the diffused LEDs is very similar, even though the material properties are different. For example, the $930 \mathrm{~nm}$ LEDs are fabricated with GaAs, while the other two diffused LEDs are fabricated with AIGaAs.

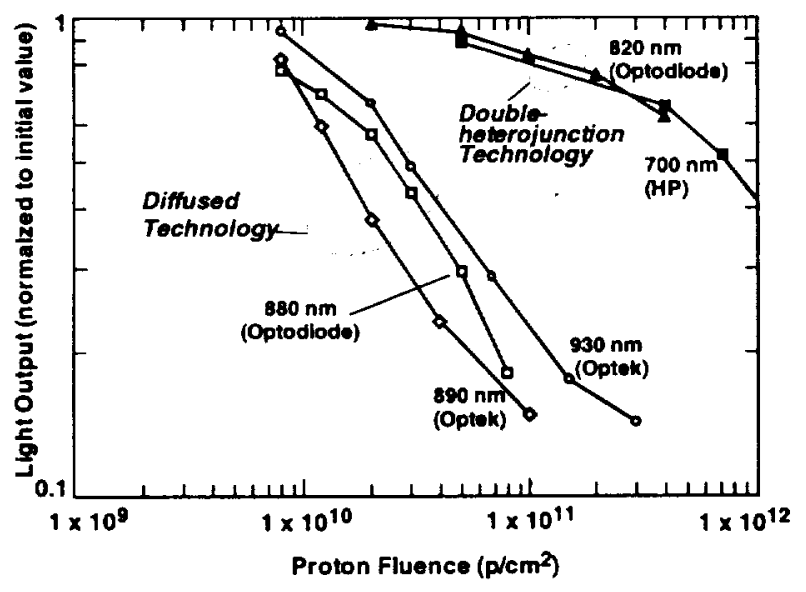

Figure 5. Degradation of several different LED technologies when they are irradiated with protons.

In addition to the difference in degradation, the two types of LED technologies also differ in the way that the damage anneals after irradiation.

Damage in the diffused LEDs is affected by current flow during or after irradiation $[2,7,10]$.

Substantial damage may recover when high operating currents are used, even for time periods of a few minutes. This is not only important for applications, but also needs to be carefully considered when doing radiation tests on LEDs or optocouplers. If measurements are extended into the high operating current region, a substantial amount of damage may anneal, reducing the apparent degradation when a series of stepped irradiation-and-measurement sequences are used. Although that may be appropriate for applications with high current, it may cause substantial underestimation of the damage that occurs with low forward current. Operating conditions during and after testing need to be carefully planned to take this dependence into account.

In contrast, damage in double-heterojunction LEDs exhibits only a very slight dependence on operating current.

\section{Phototransistor Degradation}

Linear optocouplers and some simplified digital optocouplers use basic phototransistors as detectors, although many digital optocouplers use more complex amplifier stages. Phototransistor gain degradation is one factor that contributes to CTR degradation in basic optocouplers, and that factor still remains when LEDs with improved radiation performance are used.

Figure 6 shows how protons degrade the gain of typical phototransistors. Five volts was applied between the collector and emitter during irradiation; the base region was left floating to simulate typical optocoupler applications. Measurements after irradiation were made in the conventional way, evaluating the transistor by applying a base current and measuring the resulting collector current just as for a normal transistor. One transistor is from a digital optocoupler and the other is from an analog optocoupler. In both cases very little degradation occurs until relatively high radiation levels are reached. These results were taken at a constant collector current ( $1 \mathrm{~mA}$ for the $4 \mathrm{~N} 49$, and $100 \mu \mathrm{A}$ for the transistor from the analog optocoupler). Far less degradation occurs when the phototransistors are irradiated to equivalent total dose levels with cobalt- 60 gamma rays, so that the damage is dominated by displacement effects.

Although discrete transistors are usually operated at fixed current, when phototransistors are used in an optocoupler the degradation of the LED light output will steadily reduce the operating current when the optocoupler is irradiated. Thus, the dependence of the transistor degradation on operating current must also be taken into account. 


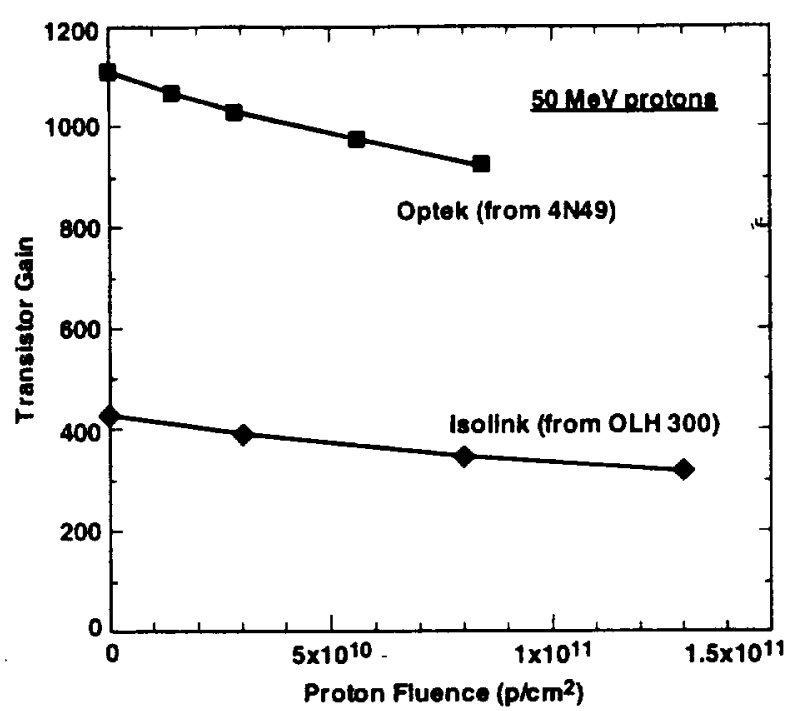

Figure 6. Gain degradation of phototransistors used in two different types of optocouplers.

Figure 7 shows how the current dependence of phototransistor gain is affected by radiation. As shown in the figure, at a fluence of $3 \times 10^{10} \mathrm{p} / \mathrm{cm}^{2}$ the decrease in gain due to the shift in operating characteristics is comparable to the decrease in gain due to radiation. This can have a significant effect on the overall degradation of CTR. For example, the optical power of the $880 \mathrm{~nm}$ LED used in the standard linear optocoupler falls by about a factor of two at $3 \times 10^{10} \mathrm{p} / \mathrm{cm}^{2}$ (see figure 1). This lowers the operating current, and increases the degradation in CTR.

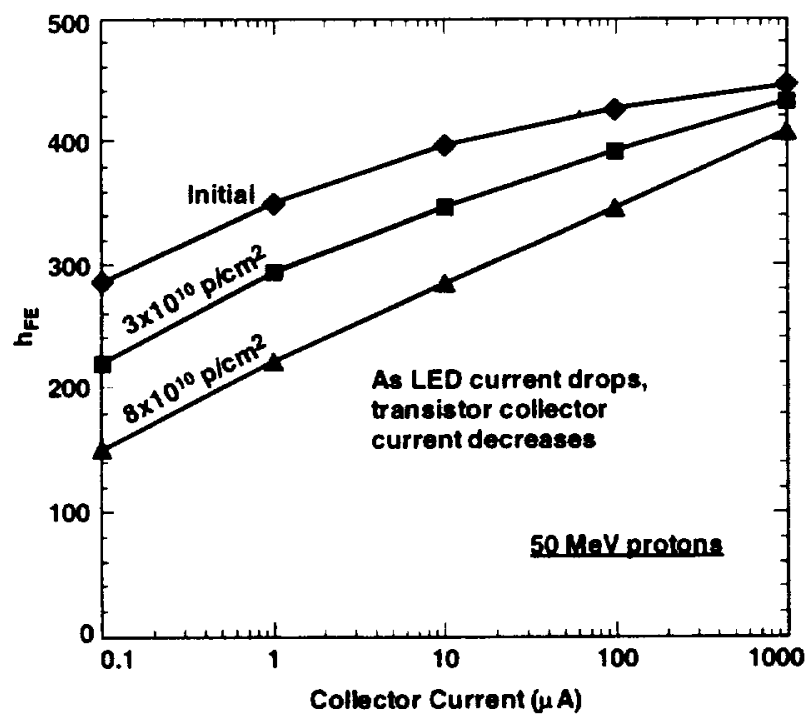

Figure 7. Dependence of phototransistor gain on operating current (transistor from an analog optocoupler process).

\section{Optical Photoresponse Degadation}

Selecting LEDs that are more resistant to radiation and modifying the detector circuit to reduce the dependence of CTR on transistor gain results in optocouplers with far less degradation to proton displacement damage than conventional optocouplers that operate at $880 \mathrm{~nm}$. However, if conventional silicon photodetectors are used, there will still be significant degradation of the optical photoresponse. Most photodetectors use a high/low p-n junction that allows some light be collected by diffusion. Photoresponse degradation is affected by wavelength because the optical absorption depth depends on wavelength (it is also affected by temperature, and that topic will be addressed in the full paper).

At $880 \mathrm{~nm}$, the " $1 / \mathrm{e}$ " absorption depth in siticon is approximately $46 \mu \mathrm{m}$, while at $700 \mathrm{~nm}$ the absorption depth is $5.6 \mu \mathrm{m}$. Some of the optical carriers will be collected within the depletion region, which is approximately $2 \mu \mathrm{m}$ for typical photodetectors with lightly doped substrates. However, a large fraction of the light is collected by diffusion. The minority carrier diffusion length $L_{n}$ in a p-substrate is

$$
\mathrm{L}_{\mathrm{n}}=[\mathrm{D} \tau]^{1 / 2}
$$

where $\mathrm{D}$ is the diffusion constant, and $\tau$ is the minority carrier lifetime. The diffusion length is reduced as the minority carrier lifetime degrades from radiation [11]. The diffusion length must be approximately three times greater than the absorption length in order to collect nearly all of the carriers. Measurable degradation occurs when the diffusion length falls below that threshold point.

Figure 8 shows the photoresponse of silicon detectors from three different optocoupler technologies (the photoresponse was measured at the wavelength used in the optocoupler application). Note that there is about an order of magnitude difference in the fluence at which significant degradation occurs, which correlates with the wavelength. The dashed lines show calculations of photoresponse based on a model for solar cell degradation [12]. There is close agreement between the calculations and experimental data for the three devices at $50 \mathrm{MeV}$. 


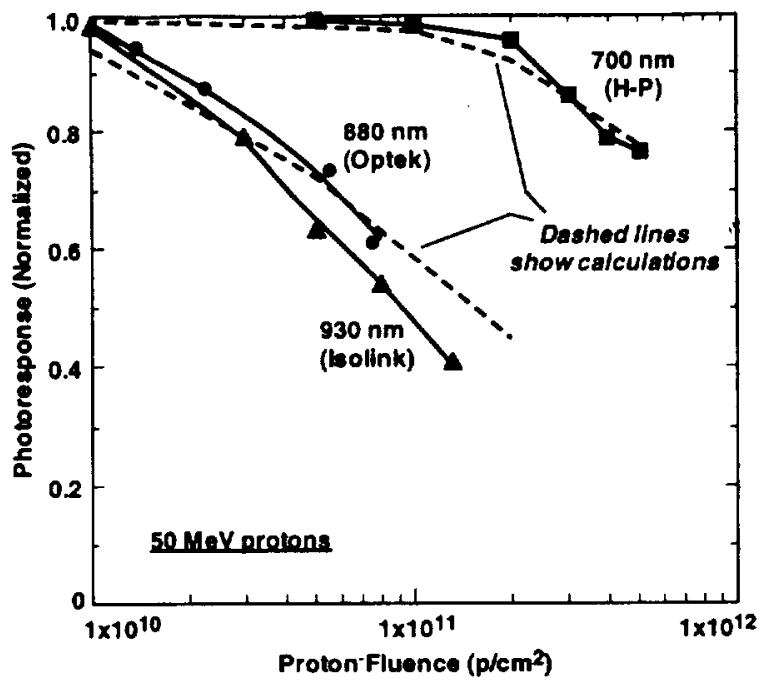

Figure 8. Degradation of optical photoresponse for detectors from three different optocoupler technologies.

Photoresponse measurements were also made on a discrete phototransistor that can be used over a wide range of wavelengths. The phototransistor was connected as a diode, measuring optically induced photocurrent in the base-collector junction. These results are shown in Figure 9. They are in general agreement with the results for detectors used in the optocouplers in Figure 8. Note that the degradation is much greater at longer wavelengths, in agreement with the assumption that the degradation is dominated by changes in minority carrier diffusion length.

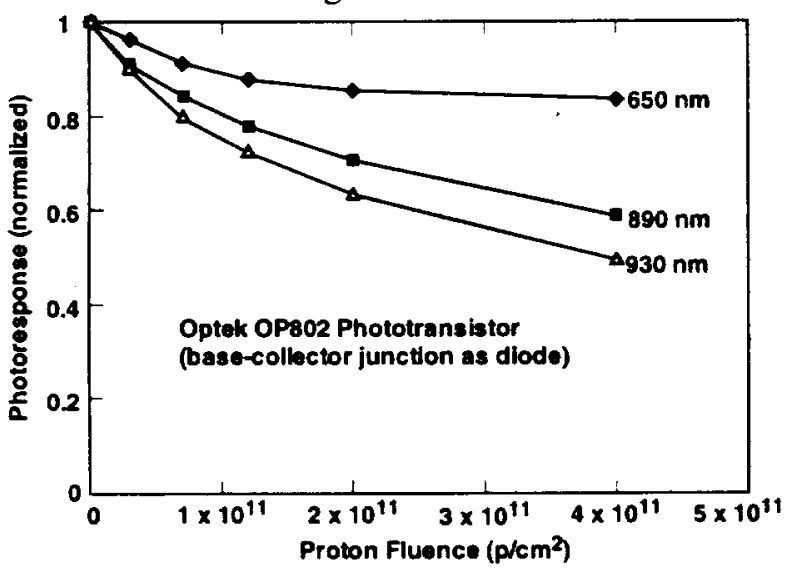

Figure 9. Degradation of the photoresponse of a generalpurpose phototransistor at various wavelengths.

The results in Figures 8 and 9 suggest that there is an upper limit to the radiation level for siliconbased detector technologies that corresponds to the inherent limitations of photoresponse. However, other silicon detectors are available which do not depend on collection of photo-induced current from diffusion. A P-I-N detector was also evaluated at various wavelengths. It was reverse biased at $-5 \mathrm{~V}$, which was sufficient to fully deplete the i-region of this device. Those results are shown in Figure 10, using the same scale as that of the phototransistor in Figure 9. Far less degradation occurs, particularly at longer wavelengths. This illustrates that alternative detector technologies can improve optocoupler performance even further, more consistent with the improved performance of double-heterojunction LEDs.

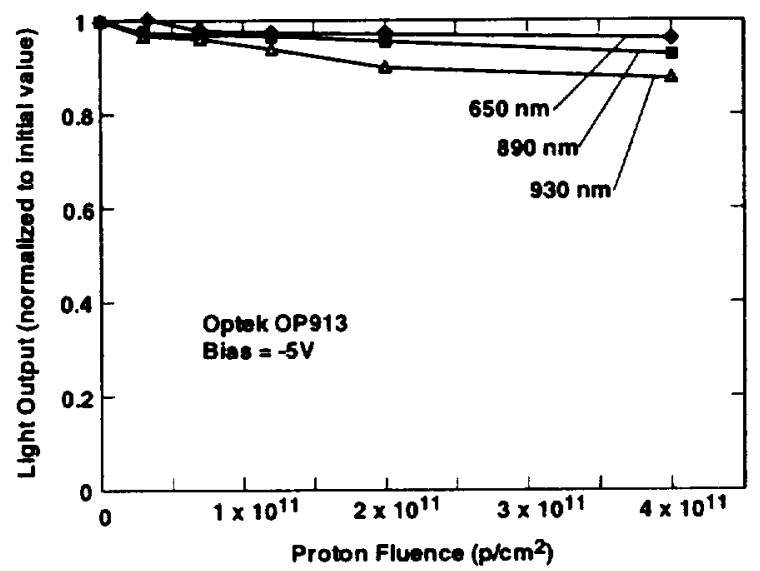

Figure 10. Degradation of the photoresponse of a P-I-N detector at various wavelengths.

\section{Discussion}

Three underlying factors contribute to optocoupler degradation. All three factors are affected by displacement damage, and it is important to do radiation tests with high-energy protons, not just gamma rays, in order to determine how optocouplers will survive in typical space environments.

The most important factor is LED degradation. As noted in earlier work [1-3], certain types of LED technologies are extremely sensitive to displacement damage. However, it is possible to select optocouplers with double-heterojunction LEDs that are inherently more resistant to displacement damage. This typically will increase the radiation hardness by an order of magnitude or more. One must keep in mind however that optocouplers are hybrid devices. The specifications usually do not include the wavelength or basic technology of the LEDs. Some manufacturers purchase LEDs from external suppliers, and have little knowledge or control of the LED technology that they use other than their operation within the electrical specifications of the overall optocoupler. 
Variations in LED technology and supplier can cause optocoupler hardness to vary over a wide range, limiting the value of archival radiation test data.

Although one would normally expect transistor gain degradation to be important, typical phototransistors in modern devices have relatively narrow base regions which reduce their sensitivity to displacement damage. They also are relatively resistant to ionization damage. Even though gain degradation is the least important factor, the current dependence of transistor gain will add to the CTR degradation as the LED output degrades. That factor is particularly important for optocouplers with sensitive LEDs because the light output decreases so much at low radiation levels.

The third factor, optical photoresponse, has not received sufficient attention. For optocouplers with improved LED hardness, optical photoresponse is the largest contributor to CTR degradation, which is evident by comparing the LED degradation in Figure 1 with photoresponse degradation in Figures 8 and 9. Photoresponse degradation depends on wavelength because the absorption coefficient is wavelength dependent. It is possible to design structures with epitaxial layers that reduce the effective absorption depth, but that also reduces the responsivity and efficiency. There are also alternative detector technologies with less degradation (see Figure 10), but it is likely that the photoresponse of conventional silicon photodetectors will be the limiting factor in the radiation performance of most optocouplers.

Radiation can also affect light transmission through the coupling material. That mechanism has not been important in any of the optocouplers that we have evaluated to date.

In summary, this paper has discussed proton degradation in two basic types of optocouplers. By selecting improved LED technologies, it is possible to use optocouplers at equivalent total dose levels of $20 \mathrm{krad}(\mathrm{Si})$ or more in environments that are dominated by protons. With improved LEDs, photoresponse degradation is the most important factor in optocoupler degradation. Optocouplers that operate at shorter wavelength are less affected by photoresponse degradation because the light is absorbed in much shallower regions. That provides an inherent advantages for optocouplers that operate in the visible range (approximately $700 \mathrm{~nm}$ ) compared to those that operate near the peak in silicon responsivity $(900 \mathrm{~nm})$.

\section{References}

1. B. G. Rax, et al., "Total Dose and Proton Damage in Optocouplers," IEEE Trans. Nucl. Sci., 43, 3167 (1996). opto paper

2. M. D'Ordine, "Proton Displacement Damage in Optocouplers," 1997 IEEE Radiation Effects Data Workshop, IEEE pub. 97TH8293, p. 122.

3. A. H. Johnston, "Proton Damage Effects in Commercial Optocouplers," collected papers from RADECS98, p. 87.

4. B. H. Rose and C. E. Barnes, "Proton Damage Effects on Light Emitting Diodes," J. Appl. Phys., 53(3), 1992 (1982).

5. C. E. Barnes, "The Effects of Radiation on Optoelectronic Devices," SPIE, 721, 18 (1986).

6. H. Lischka, et al, "Radiation Effect in Light Emitting Diodes, Laser Diodes, Photodiodes and Optocouplers," RADECS93 Proceedings, p. 226.

7. H. Lischka, et al., "Gamma and Neutron Irradiation of Optoelectronic Devices," RADECS95 Proceedings, p. 560.

8. A. L. Barry, et al., "The Energy Dependence of Lifetime Damage Constants in GaAs LEDs for 1-500 MeV Protons," IEEE Trans. Nucl. Sci., 42, 2104 (1995).

9. G. P. Summers, et al., "Displacement Damage in GaAs Structures," IEEE Trans. Nucl. Sci., 35, 1221 (1988).

10. A. H. Johnston, B. G. Rax and L. Selva, "Proton Irradiation of Light-Emitting Diodes," to be presented at the Nuclear and Space Radiation Effects Conference, Norfolk, Virginia, July, 1999.

11. G. C. Messenger and M. S. Ash, The Effects of Radiation on Electronic Systems, New York: Van Nostrand Rheinhold, 1992.

12. JPL Solar Cell Radiation Handbook, JPL Publication 8269. November, 1982. 
Viewgraphs for Presentation on Proton Damage in Linear and Digital Optocouplers

A. H. Johnston

1 - Outline

Basic Relationships

Comparison of Standard and Hardened Optocoupler Responses

Comparison of Different LED Technologies

Phototransistor Gain Degradation

Photoresponse Degradation

Conclusions

\section{2 - Basic Relationships}

Three Factors Are Important in Optocoupler Degradation

LED Output Power

Transistor or Amplifier Gain

Photoresponse

LED Degradation Often Dominates

Displacement Damage (protons)

Depends on LED Technology

Two Basic Types of Optocouplers

Digital Optocouplers

Linear Optocouplers

3 - Figure 1 from paper (Degradation of the two LED technologies used in standard and hardened optocouplers)

4 - Figure 2 from paper (Degradation of standard and special versions of the OLH300 linear optocoupler)

5 - Figure 3 from paper (Degradation of the standard version of the optocoupler at various LED forward currents)

6 - Figure 4 from paper (Degradation of the special version of the optocoupler at various LED forward currents 
7 - Figure 5 from paper (Degradation of several different LED technologies when they are irradiated with protons)

8 - Figure 6 from paper (Gain degradation of phototransistors used in two different types of optocouplers)

9 - Figure 7 from paper (Dependence of phototransistor gain on operating current; transistor from an analog optocoupler process)

\section{0 - Photoresponse}

Silicon Absorption Coefficient Depends on Wavelength

Typical Absorption Depth

- $\quad 7 \mu \mathrm{m}$ at a wavelength of $650 \mathrm{~nm}$

- $\quad 12 \mu \mathrm{m}$ at a wavelength of $800 \mathrm{~nm}$

- $\quad 60 \mu \mathrm{m}$ at a wavelength of $900 \mathrm{~nm}$

Most of the Light Is Collected by Diffusion

- Diffusion Length $\mathrm{L}=\sqrt{\mathrm{D} \tau}$, where $D$ is the diffusion constant and $\tau$ is the minority carrier lifetime

- Displacement Damage Degrades Lifetime, Reducing L

- Less Significant for Shorter Wavelengths because of Reduced Absorption Depth

11 - Figure 8 from paper (Degradation of optocoupler photoresponse for detectors from three different optocoupler technologies)

12 - Figure 9 from paper (Degradation of the photoresponse of a general-purpose phototransistor at various wavelengths)

13 - Figure 10 from paper (Degradation of the photoresponse of a P-I-N detector at various wavelengths) 


\section{3 - Conclusions}

Results for Hardened Optocoupler Show More than 10X Improvement

- Photoresponse Degradation Becomes Significant

- Current Dependence of Phototransistor also Affects Results

Double-Heterojunction LEDs Much Less Degraded by Radiation

Optocoupler Selection Techniques

- $\quad$ Analog Optocouplers Are Designed Differently

Masks Some Mechanisms

Introduces Nonlinearities in Response

- $\quad$ LED Technology Is the First-Order Problem

- Best Performance

Double-Heterojunction LEDs

Short Wavelengths

High-Gain Amplifiers 